ARCHIVOS

de historia del movimiento obrero y la izquierda
ISSN 2313-9749

ISSN en línea 2683-9601

Año IX, no 17 , pp. 163-183

septiembre de 2020-febrero de 2021

\title{
Convergencias contradictorias. Proletariado industrial y concepción programática bolchevique entre 1917 y 1929
}

\author{
Martín Alejandro Duer \\ Consejo Nacional de Investigaciones Científicas y Técnicas \\ Universidad Nacional de San Martín - Universidad de Buenos Aires \\ martin_duer85@hotmail.com
}

Titulo: Contradictory convergences. Industrial proletariat and Bolshevik programmatic conception between 1917 and 1929

Resumen: El presente trabajo indaga el carácter contradictorio que asumió la convergencia de los diversos proyectos de reconstrucción de la sociedad luego de la Revolución Rusa de 1917. Se argumentará que los consensos entre Partido Bolchevique y movimiento obrero, relativos al fortalecimiento de una instancia estatal centralizada capaz de desenvolver una planificación global, se desarrollaron en conflictiva interacción con su contracara programática de asimilación de la llamada organización científica de trabajo.

Palabras clave: bolcheviques - organización del trabajo -control obrero

\begin{abstract}
This work investigates the contradictory character assumed by the convergence of the various projects for the reconstruction of society after the Russian Revolution of 1917. It will be argued that the consensus between the Bolshevik Party and the worker's movement, regarding the strengthening of a centralized state instance capable of developing a global planning, developed in conflictive interaction with their programmatic counterpart of assimilation of the so-called scientific organization of labor.
\end{abstract}

Keywords: bolsheviks - organization of labor - worker's control

Recepción: 30 de julio de 2020. Aceptación: 4 de septiembre de 2020 


\section{Introducción}

El derrotero que condujo desde las prometedoras perspectivas emancipatorias abiertas por las revoluciones de 1917 hasta la emergencia y eventual consolidación del estalinismo hacia fines de la década de 1920, ha sido abordado desde múltiples perspectivas. Las lecturas que, durante la Guerra Fría, hegemonizaron el campo historiográfico en torno a esta problemática tendieron a inscribirla en un terreno delimitado por lógicas explicativas diametralmente opuestas. Así, el estalinismo fue concebido como el lógico corolario de los lineamientos teórico-programáticos del bolchevismo originario, o bien como una fundamental ruptura con éstos (Cohen, 1999). Luego de la disolución de la Unión Soviética, el extraordinario caudal de archivos desclasificados fue estudiado bajo el prisma de una renovada línea teórico-conceptual que, sin renegar del todo respecto de las visiones de sus predecesoras, matizaría la otrora dominante clave interpretativa situada en los términos mutuamente excluyentes de continuidad o ruptura (Fitzpatrick, 2007, pp. 88-89; Baña, 2017, pp. 227-228). Desde entonces, la aspiración hacia una ampliación del horizonte analítico implicó un abandono del clásico encorsetamiento en etapas históricas concretas -octubre de 1917, comunismo de guerra, Nueva Política Económica (NEP), estalinismo, etc.-, de modo de comprender, como oportunamente señalara Stephen Kotkin, "todo el período soviético en términos de las vicisitudes de la revolución" (1998, p. 387).

Adhiriendo a este propósito analítico, y sin pretender agotar la cuestión, el presente trabajo procura efectuar un aporte a la misma. En este sentido, se estudian los rasgos salientes de un proceso determinado por la interrelación entre los objetivos programáticos del Partido Bolchevique, relativos a las tareas de la clase obrera en la transición al socialismo, y las aspiraciones de amplias fracciones del proletariado industrial de los principales centros urbanos de la Rusia Central por consolidar sus conquistas económico-sociales bajo el resguardo de un Estado concebido como propio. La importancia analítica de esta región responde no sólo a su preponderancia económica, sino también al consenso historiográfico (Rassweiler, 1983; Brower, 1989; Koenker, 1989; Depretto, 1994) -incluyendo estudios que efectuaran en su momento los propios historiadores soviéticos (Drobizhev, 1979; Vorozheikin y Seniavskii, 1977)-, en torno a la relativa estabilidad de su población obrera entre 1917 y fines de la década de 1920, lo cual habilita a tomar como premisa la existencia de cierta continuidad de posicionamientos -tanto del Partido como de las bases obreras-durante el período considerado. 


\section{Tendencias político-organizativas hacia la producción industrial planificada y la organización científica del trabajo en la industria soviética}

En vísperas de la toma del Palacio de Invierno, Lenin concebía la subordinación de la maquinaria estatal burguesa al poder organizado del proletariado como el ineludible prerrequisito programático de la transición hacia el socialismo. De acuerdo con su perspectiva en torno a la especificidad de la época imperialista, el moderno organismo estatal expresaba palmariamente la tendencial fusión entre el capital financiero y la más abarcadora instancia de mando concebible a escala nacional sobre el conjunto de sus operaciones. A su vez, el proceso revolucionario ruso había reconstituido los soviets surgidos en 1905 que, en cuanto órganos aún embrionarios de la dictadura del proletariado, suponían la modalidad organizativa adecuada para una apropiación por las masas trabajadoras de las funciones directivas que sobre la economía podría ejercer dicha maquinaria estatal. De lo que se trataba, en consecuencia, era de proceder a una absorción por los soviets de los dispositivos de organización consciente sobre un trabajo crecientemente socializado. Así, juntamente con la destrucción de la organización específicamente opresora del Estado burgués sobre las clases explotadas, Lenin remarcaba que la tarea revolucionaria debía consistir en la apropiación del aparato estatal que, al hallarse intimamente enlazado con los bancos y los principales consorcios capitalistas, permite efectuar "un vasto trabajo de cálculo y registro": "Este aparato no puede ni debe ser destruido. Lo que hay que hacer es arrancarlo de la supeditación a los capitalistas [...] subordinarlo a los soviets proletarios y darle un carácter más amplio, más vasto y más popular" (1958, p. 94). El cambio del carácter de clase de la maquinaria estatal colocaría de inmediato, con "un solo decreto del gobierno proletario", los más avanzados métodos organizativos desarrollados por el capitalismo en manos de los trabajadores, que por ello devendrian en "funcionarios de Estado". Por lo demás, en la medida en que "el capitalismo ha simplificado las funciones de cálculo y de control, reduciéndolas a asientos relativamente sencillos, al alcance de cualquier persona que sepa leer y escribir" (p. 94), resultaría perfectamente factible para el proletariado triunfante convertir en funcionarios estatales al ejército de empleados y cuadros administrativos y técnicos -los "especialistas"-, controlando que su labor sirviera en lo sucesivo a sus intereses de clase.

A partir del triunfo revolucionario de octubre de 1917 Lenin, desde su puesto de presidente del Consejo de Comisarios del Pueblo-Sovnarkom-, encabezaría los proyectos partidarios de reorganización político-institucional en función del propósito excluyente de integrar a las masas obreras y campesinas al funcionamiento de la maquinaria estatal. Esta 
pretensión orientó el diseño del Departamento de Administración -upravleniye delami- del Sovnarkom, cuyos integrantes fueron reclutados por fuera del cuerpo administrativo zarista que aún poblaba las oficinas de los restantes comisariados del pueblo (Douds, 2018, pp. 87-89).

Se previó igualmente una modalidad paralela de integración popular al naciente Estado soviético. Desde mediados de 1918 avanzaron los proyectos de fusión de la miriada de instancias de control que hasta entonces actuaban independientemente las unas de las otras. Lenin concibió la posibilidad de conformar un "Departamento de Inspección Obrera y Campesina" que propiciara la participación en su seno de "toda la masa trabajadora", con el fin de que ésta asimilara el arte de la administración estatal (1960d, p. 297). El esquema atendía formalmente a "involucrar al pueblo en el trabajo de la administración estatal como parte de la transición al comunismo", y para ello, "el Estado habría de ser transformado y proletarizado" (Rees, 1987, p. 23). Finalmente, en virtud de un decreto del Comité Ejecutivo Central Panruso de los Soviets -VTsIK- del 7 de febrero de 1920, fue constituido el Comisariado del Pueblo para la Inspección Obrera y Campesina -NKRKI-. A él quedaron subordinados todos los organismos de inspección obrera que habian surgido hasta entonces. El NKRKI-dependiente tanto del Partido como de las máximas instancias de la autoridad del Estado-, se encargaría de controlar y supervisar la labor de los 17 comisariados restantes.

El diseño, por tanto, estaba dirigido a garantizar el comando directo de la maquinaria estatal por parte de las masas obreras y campesinas, en la medida en que éstas participaran activamente en el Comisariado del Pueblo para la Inspección Obrera y Campesina, adquiriendo por esta vía el aprendizaje necesario para semejante tarea. Concretamente, el NKRKI debía constituirse en la plataforma para el desenvolvimiento de un proceso de dos vías: al tiempo que propiciaría un efectivo control obrero sobre el trabajo de los "especialistas burgueses", aseguraria los medios para que, a través de esta función de control, las propias masas trabajadoras adquiriesen el conocimiento técnico de la intelectualidad burguesa que les permitiera a ellas mismas tomar en sus manos la dirección de "su" Estado (Lenin, 1960c, p. 349).

No obstante, de acuerdo con el diagnóstico de Lenin, la dirección obrera sobre el funcionariado burgués en el Estado constituía tan solo una de las cuestiones que planteaba la dinámica transicional. Su contracara residía en el ámbito económico. Aún cuando la República de los soviets había logrado forjar el adecuado armazón político para cimentar el socialismo, el desarrollo del capitalismo ruso alcanzado hasta entonces era insuficiente para proveer la base económica requerida como punto de partida del nuevo modo de producción. El caso diametralmente opuesto lo ofrecía Alemania. Esta nación, afirmaba Lenin, al tiempo que contaba 
con una base económica fundada en la "última palabra de la moderna técnica capitalista y de la organización planificada" de la producción, estaba gobernada por el "imperialismo junker-burgués". Por lo tanto, si en lugar del "Estado militar, junker, burgués, imperialista", gobernara sobre esta base "otro Estado, pero de tipo social diferente, de distinto contenido de clase, el Estado soviético, es decir, proletario", se obtendría "toda la suma de condiciones que dan como resultado el socialismo" (1960b, pp. 332-333). La fusión de ambas dimensiones en una única formación socioeconómica cohesionada constituiría así la premisa fundamental de la edificación socialista. Consecuentemente, mientras continuaran separadas estas "dos mitades de socialismo" debido al retraso de la revolución en Alemania, el proletariado soviético habria de conformar una base económica adecuada a su dominación política. Y ello, para Lenin, presuponía tanto el avance de la "trustificación" de empresas en las distintas ramas productivas, como la asimilación del régimen productivo que constituía la norma en el caso alemán, el propio del capitalismo monopolista de Estado.

Esta proyección programática implicaba una profunda reorganización del proceso de trabajo en la fábrica. En términos relativos, señalaba Lenin, "el ruso es un mal trabajador", de modo que debe "aprender a trabajar" de acuerdo con las más avanzadas modalidades capitalistas de organización del proceso de trabajo (1960a, p. 254). En este terreno, se destacaba el sistema diseñado por el ingeniero norteamericano Frederick Taylor el cual, "al igual que todos los progresos del capitalismo, reúne en sí toda la refinada ferocidad de la explotación burguesa y muchas valiosísimas conquistas científicas" (p. 254). "La posibilidad de realizar el socialismo", concluía, "quedará precisamente determinada por el grado en que logremos combinar el poder soviético y la forma soviética de administración con los últimos progresos del capitalismo. Hay que organizar en Rusia el estudio y la enseñanza del sistema Taylor, su experimentación y adaptación sistemáticas" (p. 255). El otrora "izquierdista" Bujarin señalaría un año después -en la obra de divulgación escrita junto con Preobrazhensky del programa aprobado en el VIII Congreso partidario, El ABC del comunismo (1922)-, que la "organización científica de la producción" constituye una premisa fundamental de la futura sociedad comunista (p. 292).

En este sentido, Lenin ya había argumentado hacia la primera mitad de 1918 que la contratación de los "especialistas burgueses" como administradores de las grandes plantas industriales a cambio de una elevada remuneración, constituía un "tributo" que temporalmente debía soportar el proletariado de modo de superar sus deficiencias en lo que respecta a la administración del proceso de trabajo de acuerdo con la más avanzada técnica capitalista. Se habría tratado no obstante de una 
medida que eventualmente demostraría su carácter beneficioso ante un proletariado políticamente dominante: "Cuanto antes aprendamos nosotros mismos, los obreros y campesinos, a tener una disciplina mejor y una técnica de trabajo más elevada, aprovechando para este aprendizaje a los especialistas burgueses, tanto más rápidamente nos libraremos de todo "tributo" a estos especialistas" (Lenin, 1960a, pp. 246-247).

A su vez, para la dirigencia bolchevique, la necesidad de la expansión de la producción fundada en la gran industria resultaba particularmente acuciante hacia comienzos de 1921. Por entonces, las devastadoras consecuencias de la guerra civil se proyectaban en un escenario marcado por un sensible descenso en los principales indicadores económicos (Davies, 2007, p. 23). El malestar generalizado entre la población, y particularmente entre el campesinado, tornaba inviable la continuidad de la política de requisas forzosas de grano para garantizar el abastecimiento de las ciudades. La extrema centralización estatal sobre la producción y la distribución característica del comunismo de guerra debía ceder el terreno. En lo sucesivo, la NEP - si bien concebida fundamentalmente para el restablecimiento del flujo mercantil entre ciudad y campo-, procuraria igualmente promover concesiones al capital privado extranjero para que éste se encargara de la explotación de ciertos sectores industriales, en auxilio de la agotada capacidad económica del Estado soviético que, por lo demás, debía hacer frente a la producción con un desgastado acervo de capital fijo. ${ }^{1}$

Aquí residía para Lenin la clave de la consolidación del capitalismo de Estado en la estructura económica rusa. La defensa de esta postura en 1921 reproducía la línea expuesta en 1918. Aún cuando el sistema de concesiones representara un "tributo" que el gran capital obtenía del régimen soviético debido a sus carencias técnicas y culturales, este último, argumentaba Lenin, obtendría un "resarcimiento" al asimilar por esta vía la organización del proceso de trabajo de acuerdo con la más avanzada técnica capitalista, el sistema Taylor (1960e, p. 340).

Internalización de los mecanismos de dirección de una maquinaria estatal capaz de planificar centralizadamente la producción social y dominio del método "científico" de organización del proceso de traba-

1. La problemática en torno a las políticas partidarias relativas al empleo intensivo de fuerza de trabajo para compensar las deficitarias existencias de maquinaria industrial luego de la Guerra Civil suscitó una amplia producción bibliográfica. Es posible destacar entre otros los trabajos de M. Gardner Clark (1956), Jean Marie Collette (1965), Maurice Dobb (1967) y Richard Day (1973). Se argumentará aquí que estas condiciones deficitarias operaron como acicate para conceder mayor centralidad a las proyecciones programáticas del Partido, concebidas durante el período inmediatamente pre-revolucionario, referentes al incremento de la productividad a partir de la sistemática implementación de la llamada organización científica del trabajo. 
jo constituyeron así las principales consignas programáticas a partir de las cuales el bolchevismo procuró orientar la dinámica del período post-revolucionario (Linhart, 1976).

\section{Realización programática, control obrero y tsekhovshchina}

La reorganización del ascendente régimen político-institucional soviético pronto reflejó en su configuración estas perspectivas programáticas. La regimentación del aparato productivo quedó formalmente expresada en el decreto del 28 de junio de 1918 sobre nacionalización de la industria a gran escala. Se pretendía, desde entonces, encuadrar las medidas de confiscación sobre la burguesía en el mentado proyecto de conformación de un capitalismo de Estado tendiente a crear las bases para el eventual avance hacia el socialismo. Así, de acuerdo con sus primeros dos artículos, las principales empresas industriales y comerciales de la República Soviética eran nacionalizadas y colocadas bajo la dirección de los correspondientes departamentos del Consejo Supremo de Economía Nacional-Vesenkha- (Daniels, 1993, p. 82). Por su parte, el artículo tercero estipulaba que las empresas nacionalizadas fueran otorgadas en concesión, sin contraprestación, a sus antiguos dueños, quienes habrian de seguir financiándolas y obteniendo las ganancias procedentes de las mismas. Por lo demás, en virtud del artículo quinto, la totalidad del personal de cada una de estas empresas -incluyendo a sus dueños y miembros de la junta directiva-, habría de constituirse en empleados del Estado Soviético. A éste le siguió una serie de decretos de nacionalización de empresas individuales. Hacia fines de 1920, la totalidad de la industria de gran escala y el $92 \%$ de la mediana industria de la Rusia Central había sido nacionalizada y colocada bajo la dirección de administraciones centrales directamente dependientes del Vesenkha. El sector nacionalizado, a su vez, comprendía al 90\% de la fuerza de trabajo del país (Malle, 1985, p. 67). Asimismo, las diversas ramas de la producción quedaban encuadradas bajo la dirección de sus correspondientes jefaturas y comités centrales -glavki y tsentry- los cuales, subordinados al Vesenkha, extendian su capacidad decisoria sobre el conjunto de empresas -tanto nacionalizadas como privadas- de su respectiva rama. Finalmente, en la misma linea se conformaba en febrero de 1921 la Comisión Estatal de Planificación General -Gosplan-, a cuyos especialistas se les encomendaría desde un inicio la confección de los planes conducentes a convertir a la Rusia soviética en una potencia industrializada (Davies y Khlevnyuk, 1997, pp. 32-33).

Un porcentaje considerable de empleados estatales fue reclutado entre las filas del proletariado. No obstante, esta mayor importancia cuantitativa no implicó que los trabajadores lograsen abstraerse de la 
lógica burocrática característica del funcionamiento del Estado. Antes bien, al contrario. Los empleados de cuello blanco -sluzhashchiye-, surgidos de los estratos sociales medios, adquirieron una preponderancia descollante a partir de 1917 como organizadores en los soviets, los ministerios devenidos en comisariados, los consejos económicos, los sindicatos e incluso en el propio Partido Comunista. Ciertamente, la dirigencia bolchevique pretendía lograr la proletarización de estos elementos que, como experimentados funcionarios de los anteriores organismos burocráticos, se convertirian en una pieza clave del armado y funcionamiento del nuevo Estado soviético. Sin embargo, fue este personal reciclado quien instruyó a los obreros de cuello azul con sus propios modelos de organización jerárquica y sus patrones de trabajo (Orlovsky, 1989, pp. 190, 201-202). Los proyectos tendientes a imponer la dirección obrera sobre los cuadros administrativos derivaron en el incremento en el caudal de trabajadores comunistas integrando las dependencias estatales, pero ello no impidió la eventual subsunción de éstos a la dinámica burocrática (Douds, 2018, pp. 39-41, 107-111).

Un desenvolvimiento semejante se constata en la reorganización de la estructura industrial. La integración de los trabajadores fabriles se extendió desde las células del partido, los comités de fábrica y los sindicatos hasta los más encumbrados niveles gerenciales. Los "directores comunistas" de los trusts industriales sintetizaron el proceso de ascenso social: surgidos en su mayoría de las filas obreras, se previó que la incorporación de esta nueva camada directiva, durante y después de la guerra civil, anulara la influencia de los viejos gerentes y especialistas burgueses (Pirani, 2006, p. 204; Barber y Davies, 1994, pp. 83-84).

Esta profunda reorganización global se desplegó sobre un horizonte social atravesado por la organización y movilización revolucionarias de la población trabajadora. Desde 1916, se intensificó la actividad de agitación en las fábricas en torno a las condiciones de vida y de trabajo de los obreros de los principales centros industriales del país. La Revolución de Febrero desató las energías hasta entonces contenidas del activismo obrero. La eliminación de las restricciones a la sindicalización y a la formación de comités fabriles derivó en el inmediato encuadre orgánico de amplios sectores del proletariado urbano, motivado primordialmente por el objetivo de salvaguardar las recientemente obtenidas conquistas económicas, laborales y sociales.

Por su parte, destacados referentes de la historia social abordaron los procesos de control obrero desplegados a lo largo de 1917 en los lugares de trabajo. Surge de estos abordajes una dinámica característica en el accionar de los obreros de los principales centros industrializados. Organizados en sus respectivos comités de fábrica, éstos fueron avanzando desde contemporizadores posicionamientos de colaboración con 
el estrato gerencial, con el fin de mantener la actividad productiva en cada planta, hasta la convicción acerca de la necesidad de ampliar su participación en la administración de la empresa, supervisando cuestiones relativas a la disciplina, al despido y contratación, a los ritmos de trabajo, a la obtención de suministros y comercialización de los productos. Estos autores remarcaron igualmente la existencia de una voluntad por coordinar globalmente las múltiples actividades directivas ejercidas en el ámbito local a través de una instancia centralizadora bajo el amparo de un gobierno socialista (Koenker, 1981; Mandel, 2018 [1983]; Smith, 1985; Rosenberg, 1985 y 1994; Marot, 2012).

Sin embargo, a pesar de esta amplitud de perspectivas en torno a los intereses generales de la clase, la organización obrera estuvo igualmente atravesada por una fuerte tendencia a la resolución local de las más inmediatas dificultades surgidas del lugar de trabajo particular. Los propios líderes del movimiento obrero empleaban el término tsekhouschina-que podría traducirse como "tallerismo", "orientación hacia el taller" o bien, "identidad a partir del oficio"- para referirse a este sectarismo ocupacional entre los trabajadores (Rosenberg, 1978, pp. 93-94; Sirianni, 1985, pp. 70, 72). Esta inclinación hacia el particularismo fabril se evidenció fundamentalmente en la miriada de organizaciones laborales que emergieron en Rusia desde los primeros meses de 1917. Los comités de fábrica que, a través de sus delegados elegidos por las bases, ejercían las mencionadas tareas de control y supervisión sobre el estrato gerencial -o bien de gestión directa cuando los dueños y el personal administrativo decidían abandonar el negocio-, se distinguían según las unidades productivas a las que pertenecieran. Igualmente, la identificación obrera tendía a afianzarse más estrechamente de acuerdo con los distintos rubros en que se subdividiera el trabajo dentro de la propia fábrica. Entre otros, es el caso de la emblemática planta Putilov, donde la vinculación con cada especialización sectorial tendía a prevalecer como rasgo identitario sobre el reconocimiento de la condición de trabajador común a todos los operarios del mismo complejo industrial (Rosenberg, 1978, p. 94).

La tsekhouschina también se constata en el plano sindical. El súbito resurgimiento de las estructuras sindicales durante los primeros meses de 1917 desembocó en la conformación de entre 60 y más de 70 organizaciones hacia fines de abril en Moscú, al tiempo que para entonces era posible contabilizar más de 110 en Petrogrado (Koenker y Rosenberg, 1989, p. 108; Rosenberg, 1978, p. 94). Aún cuando se procuró -y eventualmente se logró- establecer una organización nacional por rama de producción, se impuso un criterio de integración sindical estrechamente ligado a las diversas especializaciones dentro de cada oficio, como lo demuestra particularmente el caso de los trabajadores 
de imprenta, de la alimentación, los textiles y ferroviarios (Koenker y Rosenberg, 1989, p. 109). Más elocuente aún resulta el caso de los obreros metalúrgicos de Petrogrado, de quienes se llegó a calcular que, en mayo de 1917, participaban en aproximadamente 24 sindicatos locales (Rosenberg, 1978, p. 94).

Esta dispersión organizativa suponía un obstáculo para las pretensiones centralizadoras del naciente gobierno. En 1918, la IV Conferencia de Líderes Sindicales -de mayoría bolchevique- procuró contrarrestar este particularismo, consagrando formalmente la integración de los múltiples sindicatos en un único organismo colegiado subsumido al Comisariado del Trabajo. Aducían al respecto los delegados que, bajo el amparo de un gobierno obrero, esta unificación procuraba evitar las "perniciosas divergencias en la solución de cuestiones económicas", por lo que, añadian, "las organizaciones políticas del proletariado deben abandonar, de una vez por todas, toda acción independiente, y abolir para siempre todas las "secciones", "burós", "comisiones", etc." (Rezolyutsiya chetvertoy..., 1918, pp. 13-14).

De todas formas, el partido gobernante reconocería a los sindicatos como los responsables de la "organización de la producción en la época de la dictadura del proletariado" (Bujarin y Preobrazhensky, 1922, p. 278). Igualmente, destacaría la tarea organizativa desplegada por los comités de fábrica -reorganizados como células del correspondiente sindicato- en el ámbito de la planta. Elegidos por los obreros de una fábrica o taller, estos comités "controlan todas las cuestiones dentro de la empresa concernientes a la fuerza de trabajo", siendo responsables de la contratación y despido de trabajadores, el cuidado de las familias de los obreros de la empresa, la supervisión del pago, regulación de horas de trabajo, asuntos disciplinarios, entre otras tareas (p. 282). La confirmación partidaria de la labor de los comités de fábrica en el ámbito fabril puede interpretarse en parte como reconocimiento de una posición consolidada, en parte atendiendo a los propósitos de mantenimiento de un funcionamiento eficiente en el ámbito de la planta. De cualquier manera, expresa la conservación, en estos primeros años del período post-revolucionario, de cierto poder de determinación por parte de las organizaciones obreras y, por extensión, en mayor o menor medida, de sus representados en los lugares de trabajo. Entre otras cuestiones vitales, la designación y estabilidad en el puesto de los "directores comunistas" dependía de la aprobación de los sindicatos y de los comités de fábrica y, en medida nada despreciable, de la aquiescencia de los propios obreros de la planta. Por lo demás, los comités de fábrica desempeñaron un rol central en la organización laboral y en la cotidianidad de los trabajadores a lo largo de la década de 1920 (Sirianni, 1985, pp. 83-84).

Centralización estatal y tsekhouschina convergian eclécticamente en 
el horizonte ideológico de estas organizaciones obreras y su interrelación condicionaría el específico desenvolvimiento industrial en los centros urbanos soviéticos bajo la NEP.

\section{Agitación obrera y desarrollo económico durante la década de 1920}

Sobre la base de este específico trasfondo se desenvolvieron a lo largo de la década de 1920 tendencias divergentes. De los archivos del Directorio Político Unificado del Estado -OGPU- se desprende que, entre los años 1921 y 1929, la cantidad de arrestos ascendió a 1.004 .956 casos, de los cuales 646.864 -el 64,37\%-fueron catalogados como "crímenes contrarrevolucionarios" y "agitación antisoviética" (Getty y Naumov, 1999 , p. 588). Del mismo modo, la actividad huelguística fue intensa durante todo el período.

Paralelamente, otros datos denotan una progresiva consecución de los objetivos programáticos de mayor alcance del partido gobernante. Durante la primera mitad de la década de 1920, la tasa de crecimiento industrial fue inesperadamente elevada. De acuerdo con las cifras oficiales, entre los años 1926 y 1927 la capacidad de la industria superaba en cuatro puntos porcentuales a la registrada en 1913, mientras que en 1920 tan sólo llegaba al 20\% de aquella magnitud (Davies, 1994, pp. 135-136). Más significativo aún fue el fortalecimiento de la línea partidaria de la planificación centralizada en el Gosplan. Las posiciones más moderadas dentro de la Comisión, inclinadas a supeditar el desarrollo industrial al mantenimiento de la estabilidad monetaria a partir de la paridad con el patrón oro, fueron cediendo terreno desde 1926, primero ante los más conservadores defensores de una "industrialización planificada a través del mercado" y, a partir de 1927, frente a los impulsores de una "industrialización planificada superando al mercado" (Davies y Khlevnyuk, 1997, p. 37). En este último año se estableció por decreto la eliminación de la utilidad económica como criterio para el funcionamiento de los trusts, priorizando en su lugar el cumplimiento de los objetivos de producción fijados por el Estado (Brovkin, 1998, p. 180). A pesar del trasfondo de intensa conflictividad social, quedaban afianzadas las bases del mecanismo de la planificación centralizada de la producción que, con sus objetivos de desarrollo cuantitativo basados en balances materiales y blandas restricciones presupuestarias para su consecución, caracterizaria el proceso de desarrollo industrial de las décadas siguientes (Allen, 2003, pp. 91-110). 


\section{Racionalización, agitación, adaptación}

¿Cómo interpretar estos movimientos a la luz de las inclinaciones obreras hacia la centralización estatal y al particularismo en el taller expuestas más arriba? En primer término, debe remarcarse que, fomentada desde los primeros meses posteriores a octubre de 1917, la promoción de amplias fracciones del proletariado industrial y de los sluzhashchiye a puestos jerárquicos en la administración estatal y en la gerencia industrial creó las condiciones objetivas para la emergencia de una alianza entre estos sectores sociales, centrada en el sostenimiento y fortalecimiento de la naciente maquinaria estatal (Lewin, 1985, pp. 239-243). De mayor gravitación resultó el apoyo tácito a los contornos más generales del orden post-revolucionario por parte de vastos sectores del proletariado que permanecieron en el ámbito de la producción industrial (Rosenberg, 1985, pp. 251-252). Ello se reflejó principalmente en la amplia predisposición de estos sectores a aceptar los mecanismos institucionales de resolución de conflictos para obtener respuestas a sus reclamos (Murphy, 2008, pp. 177-180; Koenker, 2005, pp. 135-138).

Por otra parte, los imperativos de reducción de costos, aumento de la productividad e intensidad del trabajo y maximización de utilidades que dictaron las nuevas pautas de funcionamiento de las grandes plantas industriales desde comienzos de la NEP generaron los esperables efectos de caída en la tasa de crecimiento del empleo urbano. Los niveles de desocupación -cuyas cifras en 1926 rondaban entre 1 y 1,4 millones de trabajadores-, aumentaron desde la segunda mitad de la década de 1920, agravándose el cuadro en virtud de la presión ejercida por la creciente migración de fuerza de trabajo del campo a la ciudad, en una proporción mucho mayor que la registrada en el período previo a la guerra (Barber y Davies, 1994, pp. 84-86). El mayor desempleo potenció los reclamos de las diversas fracciones obreras en rechazo de los mecanismos capitalistas en los que incurría la NEP, exigiendo el fortalecimiento de la capacidad estatal por comandar un proceso planificado de desarrollo abstraído de las fuerzas anárquicas del mercado. Estos reclamos hallaron eco en el ejército de funcionarios de cuello blanco procedente de los estratos medios, para quienes la dirección consciente y el control estatal representaban el necesario resguardo frente a las ciegas y espontáneas determinaciones económicas que parecian minar las conquistas revolucionarias de 1917 (Orlovsky, 1994, p. 246). Es posible interpretar esta objetiva convergencia de clase como la base social detrás de los avances de la línea planificadora en el Gosplan analizada más arriba.

Debe atenderse igualmente a otros factores estructurales de primer orden. La tasa de crecimiento de la industria a gran escala de los períodos 
1924-1925 y 1925-1926 había superado toda expectativa de desarro1lo. Esta considerable reconfiguración del panorama económico sirvió como plataforma para que la dirigencia partidaria proyectara objetivos de mayor alcance que las preocupaciones inmediatas por recuperar los niveles previos a la guerra. En una carta escrita a Molotov en julio de 1925, Stalin establecía como prioridades avanzar en la renovación de la gastada maquinaria de la gran industria, la instalación de plantas dedicadas a la producción de herramientas con las que equipar a las explotaciones agrícolas sin depender para ello del comercio exterior y, finalmente, la expansión de la industria militar. Sin estos factores, argumentaba, las potencias capitalistas "nos aplastarán con sus propias manos" (Lih, Naumov y Khlevniuk,1995, p. 86). Sin embargo, estas ambiciosas proyecciones pronto se toparon con un limite objetivo: la tasa de crecimiento del período 1926-1927 se contrajo dado que para entonces se había absorbido el grueso de la capacidad industrial instalada (Davies, 1994, p. 136). En lo sucesivo, el crecimiento a tasas elevadas habría de sostenerse a través de una explotación intensiva de los recursos propios. En el plano industrial, ello implicaba el incremento de la productividad a partir de una intensificación del proceso de trabajo. El apoyo a esta tendencia productivista se reforzaria al interior de la dirigencia partidaria y estatal en los años siguientes, cuando la Gran Depresión dislocara la histórica modalidad de integración de Rusia en el mercado mundial como proveedora de bienes agrícolas (Sánchez-Sibony, 2014; 2019). En igual sentido operaría la percepción de la amenaza de una eventual intervención militar de las potencias imperialistas sobre la Unión Soviética, temida como esperable reacción a la crisis capitalista (Davies, 1998, p. 234).

La ofensiva a partir de esta línea productivista, por cierto, no suponía una improvisación motivada por factores circunstanciales. La mencionada alianza entre obreros ascendidos a puestos administrativos y/o gerenciales y los estratos medios de sluzhashchiye y "especialistas burgueses", propició la adhesión entre sus filas a los objetivos programáticos del partido gobernante relativos al desarrollo de la productividad del trabajo. El obrero metalúrgico y dirigente sindical, Aleksei Gastev, expresó palmariamente esta tendencia. Ferviente adepto de los métodos de estandarización de las tareas y de pagos diferenciales por rendimientos superiores o inferiores al promedio establecido de productividad, propios del taylorismo, Gastev fue un destacado promotor de la implementación -por especialistas externos al personal obrero de la fábrica- de la llamada "organización científica del trabajo" (Bailes, 1977, pp. 381-382). Bajo la dirección de Gastev, el Instituto Central del Trabajo, creado en 1920 con la entusiasta aprobación de Lenin, desarrollaría desde entonces los experimentos tendientes a perfeccionar la 
descomposición y estandarización de las diversas operaciones laborales del obrero. Los mecanismos de intensificación del trabajo resultantes de estas investigaciones, conjuntamente con los estímulos salariales para la consecución de mayores niveles de productividad de los trabajadores fueron formalmente reconocidos en marzo de $1924^{2}$ como los medios idóneos a adoptar en la administración de las fábricas para avanzar hacia la industrialización del pais. ${ }^{3}$ Debe destacarse igualmente que el proyecto de "reconstrucción" -basado en la renovación tecnológica del equipo industrial y el consiguiente incremento de la producción-, previsto por el prominente dirigente del Vesenkha, Georgii Piatakov, como el primordial objetivo de inversión de la segunda mitad de la década, tomaba como modelo a los principales centros industrializados de Occidente, Alemania y Estados Unidos. Ello incluia, ciertamente, a la administración científica del trabajo (Graziosi, 1991, pp. 559-563). Esto conduce a analizar la contracara del proceso de fortalecimiento del Estado soviético.

Desde 1925, Partido, Estado y sindicatos se embarcaron en una campaña de racionalización tendiente a lograr el necesario incremento de la productividad y la intensidad del trabajo (Brunnbauer, 2000, p. 489). Resulta elocuente la correlación entre este renovado impulso productivista y el incremento -relativo y absoluto- de huelgas motivadas por reclamos en torno a las condiciones laborales frente a las acciones motorizadas por cuestiones meramente salariales.

La cantidad de medidas de fuerza por condiciones laborales se elevó sistemáticamente a lo largo de la segunda mitad de la década de 1920, pasando de tan sólo 9 casos en 1924 a 80 en 1929. También se produjo un aumento porcentual de las mismas: de representar un 3\% del total de casos en 1924, se mantuvo en los años siguientes por encima del

2. En la segunda conferencia de toda la Unión sobre administración científica, celebrada ese mes, la línea de Gastev se impuso sobre el proyecto alternativo de organización científica del trabajo propuesto en clave de revolución cultural por la "Liga del Tiempo", encabezada por el militante bolchevique y ex miembro del Proletkult, Platon Kerzhentsev. Pese a su pretensión de constituir un movimiento desde las bases, la Liga reclutó sus miembros mayoritariamente entre los estratos medios de especialistas y técnicos, siendo minoritaria la participación en sus filas de obreros industriales. Resultado por lo demás esperable, en la medida en que, al igual que sus rivales en el Instituto Central del Trabajo, el movimiento de Kerzhentsev buscaba incrementar la productividad intensificando los rendimientos laborales (Brunnbauer, 2000; Sgrazzutti y Oliva, 2017).

3. Como al respecto señaló Kendall Bailes, Gastev sería convocado en 1936 por el entonces comisario de industria pesada, Grigori "Sergo" Ordzhonikidze para organizar a los cuadros del movimiento stajanovista. Asimismo, calcula que hacia 1938 cerca de un millón de obreros industriales habían sido formados con las habilidades laborales enseñadas en el Instituto Central del Trabajo (Bailes, 1977, p. 393). 
Cuadro 1. Causas de huelgas en la Unión Soviética, 1923-1929

\begin{tabular}{|c|c|c|c|c|c|c|}
\hline Año & $\begin{array}{c}\text { Por } \\
\text { salario }\end{array}$ & $\begin{array}{c}\text { Demora } \\
\text { en los pagos }\end{array}$ & $\begin{array}{l}\text { Condiciones } \\
\text { laborales }\end{array}$ & $\begin{array}{l}\text { Otras } \\
\text { causas }\end{array}$ & $\begin{array}{l}\text { Total con } \\
\text { datos }\end{array}$ & $\underset{\text { datos }}{\text { Sin }}$ \\
\hline 1923 & $101 \quad(32,4 \%)$ & $156 \quad(50 \%)$ & No disponible & $55 \quad(17,6 \%)$ & $71,9 \%$ & $28,1 \%$ \\
\hline 1924 & $151 \quad(50,3 \%)$ & $120 \quad(40 \%)$ & $(3 \%)$ & $20 \quad(6,7 \%)$ & $100 \%$ & $0 \%$ \\
\hline 1925 & $80 \quad(59,7 \%)$ & $20 \quad(14,9 \%)$ & $17 \quad(12,7 \%)$ & $17 \quad(12,7 \%)$ & $30,9 \%$ & $69,1 \%$ \\
\hline 1926 & $508 \quad(60,8 \%)$ & $173 \quad(20,7 \%)$ & $48 \quad(5,7 \%)$ & $106 \quad(12,7 \%)$ & $99,1 \%$ & $0,9 \%$ \\
\hline 1927 & $478 \quad(64,9 \%)$ & $75 \quad(10,2 \%)$ & $57 \quad(7,7 \%)$ & $127 \quad(17,2 \%)$ & $81,4 \%$ & $18,6 \%$ \\
\hline 1928 & $(70 \%)$ & $(7 \%)$ & $72 \quad(8,6 \%)$ & $122(14,5 \%)$ & $100 \%$ & $0 \%$ \\
\hline 1929 & $(68 \%)$ & $(5,2 \%)$ & $80 \quad(10,9 \%)$ & $117 \quad(15,9 \%)$ & $100 \%$ & $0 \%$ \\
\hline
\end{tabular}

Fuente: Murphy, 2008, p. 175.

5,7\% hasta el final de la década, alcanzando picos de $12,7 \%$ en 1925 y de $10,9 \%$ en 1929 . Cabe añadir que se ha advertido un cambio de carácter de la movilización del proletariado urbano durante los años de la NEP tardía: a diferencia del tenor ofensivo y de la masividad del accionar obrero de la primera mitad de la década de 1920 por mejoras generalizadas en las condiciones económicas, el movimiento huelguístico de este último período expresaría una reacción esencialmente defensiva ante los intentos por reducir salarios y elevar la intensidad del trabajo (Pospielovsky, 1997, p. 26; Murphy, 2008, pp. 183-188). La prolífica producción historiográfica de los últimos años permite individualizar el lenguaje de las cifras. Los estudios en torno a los trabajadores de imprenta (Koenker, 2005), a los obreros metalúrgicos (Murphy, 2005), a los trabajadores textiles (Ward, 1990; Rossman, 2005), entre otros, coinciden en señalar la resistencia al avance de la línea productivista en el lugar de trabajo.

El estudio de las cifras sobre las huelgas del período permite extraer consideraciones adicionales.

Como refleja el cuadro 2 (en página siguiente), a partir de 1925 aumentó sensiblemente el número de huelgas, pero decreció en igual medida la cantidad de huelguistas involucrados en ellas, revelando la mayor preponderancia de la iniciativa sectorial en detrimento de la solidaridad inter -e intra-fabril (Murphy, 2008, p. 184). Es posible leer esta tendencia como el reforzamiento de la tsekhoushchina, esto es, del marco en el que originariamente se desenvolvió a nivel local un control obrero sobre las propias condiciones de la producción que ahora pasaba a ser sistemáticamente desmantelado por el avance, desde afuera, de la linea productivista oficial.

Paralelamente a la confrontación abierta, se desarrollaron en el 
Cuadro 2. Actividad huelguística en la Unión Soviética, 1922-1929

\begin{tabular}{|l|c|c|c|c|}
\hline Año & Huelgas & Huelguistas & $\begin{array}{c}\text { Cantidad promedio de } \\
\text { huelguistas por huelga }\end{array}$ & $\begin{array}{c}\text { Días de trabajo } \\
\text { perdidos }\end{array}$ \\
\hline 1922 & 538 & 197.022 & 366 & No disponible \\
\hline 1923 & 434 & 168.864 & 380 & No disponible \\
\hline 1924 & 300 & No disponible & No disponible & No disponible \\
\hline 1925 & 434 & 73.243 & 169 & 91.517 \\
\hline 1926 & 843 & 106.044 & 126 & 143.730 \\
\hline 1927 & 905 & 80.784 & 89 & No disponible \\
\hline 1928 & 842 & 93.835 & 111 & 134.875 \\
\hline 1929 & 735 & 65.443 & 89 & 95.424 \\
\hline
\end{tabular}

Fuente: Murphy, 2008, pp. 172-173.

seno del movimiento obrero estrategias de adaptación que procuraron valerse de las contradicciones inmanentes a la "posición ambigua" del proletariado en el orden soviético, en cuanto clase "tanto subalterna como privilegiada" (Depretto, 1994, p. 63). Menos riesgoso y más efectivo que las acciones de fuerza resultaba dirigirse a las comisiones institucionalmente establecidas con demandas individuales de aumento salarial en base a "méritos socialistas" como la propia calificación y el trabajo duro en la fábrica (Koenker, 2005, p. 138), o bien, escudarse en la terminología oficial para denunciar a gerentes y funcionarios del Partido como "saboteadores" o "enemigos escondidos" (Brovkin, 1998, p. 189). El despliegue de estas estrategias, sin embargo, no excluye un considerable grado de internalización de estos valores entre los propios demandantes. El "hablar bolchevique", que Stephen Kotkin (1995) identificó como un rasgo característico de la "civilización estalinista" durante la década de 1930, se hallaba plenamente extendido en la Unión Soviética de la NEP. Debe destacarse, no obstante, que esta línea no fue uniformemente seguida entre las filas obreras, lo cual remite a un criterio adicional de fragmentación de la clase, relativo a las condiciones diferenciales determinadas por calificación, antigüedad, sexo, origen rural o urbano, entre otros factores. Así, durante los últimos años de la década de 1920, la predisposición a la confrontación directa era mayor entre los otkhodniki-trabajadores estacionales-y entre sectores vulnerables como el caso de las obreras textiles, frente a la mayor reticencia a la lucha abierta demostrada por rubros mejor posicionados como el de los metalúrgicos (Murphy, 2008, p. 182).

Cabe remarcar, finalmente, que el impulso de la racionalización de la 
producción en el lugar de trabajo no derivó en el esperado incremento de la productividad. En el marco de la onceava sesión del plenario que el Comité Central del Partido Comunista celebró el 10 de julio de 1928, Molotov remarcó que el fortalecimiento del Estado que garantiza el dominio político del proletariado como clase $-\mathrm{y}$, con ello, la propia "causa de la construcción del socialismo"-, descansa sobre la disposición de los obreros a comprometerse en la "elevación de la productividad laboral y en la mejora de la intensidad de su trabajo" (Danilov et al., 2000, pp. 397-398). A la entusiasta alocución de Molotov contestó escuetamente Rykov que sí se observaba la mayor intensidad impuesta al trabajador, pero que ello no se expresaba en una mayor productividad (p. 398). Rasgo que perduró aún después del Gran Viraje industrializador del ascendente estalinismo (Filtzer, 1993). Aún más elocuente en este sentido que las constantemente renovadas campañas de incremento de la productividad del trabajo -trabajo de choque, emulación socialista, stajanovismo- durante la década de 1930, resulta el reconocimiento del propio Stalin de esta impotencia al promediar el desarrollo del Primer Plan Quinquenal. En una carta escrita el 28 de septiembre de 1930, señalaba a Molotov que entre las filas obreras tan sólo una ínfima minoria de trabajadores opera "honestamente" observando los principios de la disciplina laboral y de la "emulación socialista", mientras que la mayoría son "irresponsables, ausentistas y transitorios" (Lih, Naumov y Khlevniuk, 1995, p. 219).

\section{Conclusión}

La convergencia entre el Partido Bolchevique y el movimiento obrero de los centros urbanos industrializados, relativa a la inmediata conformación de un Estado capaz de comandar un proceso de planificación de la producción social superando las anárquicas determinaciones del mercado, no tardó en revelar su arista contradictoria. En cuanto la emergente maquinaria estatal soviética logró edificarse sobre bases sociales sólidas, su dirigencia se halló en condiciones de realizar el objetivo programático de racionalizar la producción industrial de acuerdo con los métodos de la organización científica del trabajo. El desgaste del relativamente pobre acervo de capital fijo y una situación internacional interpretada como hostil propiciaron, desde mediados de la década de 1920 , un avance acelerado en torno a la consecución de dicho objetivo. Pero ello implicó una intromisión de la instancia central sobre las condiciones locales de control obrero del proceso de trabajo, conquista fundamental de la Revolución al que las diversas fracciones del proletariado industrial no renunciarian sin una batalla velada o abierta. Sobre esta lógica contradictoria se desenvolvió una dinámica relacional 
fundada en la persecución de objetivos dificilmente armonizables. Leído en la clave interpretativa propuesta aquí, el devenir revolucionario que desde 1917 se extiende a los primeros años de la década de 1930, pierde todo carácter unilineal de mera continuidad con el originario legado ideológico del bolchevismo o bien de radical ruptura con éste, para asumir su dimensión procesual.

\section{Referencias}

Allen, R.C. (2003). Farm to factory. A Reinterpretation of the Soviet Industrial Revolution. Princeton University Press.

Baña, M. (2017). La Revolución Rusa en su Centenario. Perspectivas temáticas y narrativas historiográficas. Politicas de la Memoria. Anuario de Investigación e Información del CeDInCI, $\mathrm{n}^{\circ}$ 17, 224-237.

Bailes, K. (1977). Alexei Gastev and the Soviet Controversy Over Taylorism, 1918-24. Soviet Studies, 29 (3), 373-394. DOI: $h t t p: / / d x . d o i . o r g / 10.1080 /$ 09668137708411134.

Barber, J.D., y Davies, R.W. (1994). Employment and industrial labour. En R.W. Davies, M. Harrison, S.G. Wheatcroft (eds.). The economic transformation of the Soviet Union, 1913-1945 (pp. 81-105). Cambridge University Press.

Brovkin, V. (1998). Russia after Lenin. Politics, culture and society. Routledge. Brower, D. (1989). "The city in danger": The Civil War and the Russian urban population. En D. Koenker, W. Rosenberg, y R.G. Suny (eds.), Party, State, and society in the Russian civil war. Explorations in social history (pp. 58-80). Indiana University Press.

Brunnbauer, U (2000). The League of Time (Liga Vremia). Problems of making a Soviet working class in the 1920s. Russian History, 27 (1), 461-495. DOI: $101163 / 187633100 x 00155$.

Bujarin, N. y Preobrazhensky, E. (1922). Program of the Communist Party of Russia. Adopted at the Eighth Party Congress, held march 18 to 23, 1919. En The ABC of communism (pp. 373-402). The Communist Party of Great Britain.

Clark, M.G. (1956). The economics of Soviet steel. Cambridge University Press.

Cohen, S. (1999). Bolshevism and stalinism. En R.C. Tucker (ed.). Stalinism. Essays in historical interpretation (pp. 3-29). Transaction Publishers.

Collette, J.M. (1965). Politique des investissements et calcul économique, Cujas.

Daniels, R.V. (1993). A documentary history of communism in Russia. From Lenin to Gorbachev. University Press of New England.

Danilov, V.P. et al. (2000). Kak lomali NEP. Stenogrammy plenumov TsK $V K P(b)$ 1928-1929 gg. Tomo II. Mezhdunarodnyy Fond "Demokratiya".

Davies, R.W. (1994). Industry. En R.W. Davies, M. Harrison, S.G. Wheatcroft 
(eds.). The economic transformation of the Soviet Union, 1913-1945 (pp. 131-157). Cambridge University Press.

- (1998). The industrialisation of Soviet Russia 3: The Soviet economy in turmoil, 1929-1930. Macmillan Press LTD.

- (2007). Soviet economic development from Lenin to Khrushchev. Cambridge University Press.

Davies, R.W. y Khlevnyuk, O. (1997). Gosplan. En E.A. Rees (ed.). Decision-Making in the Stalinist command economy, 1932-1937 (pp. 32-66). Macmillan Press.

Day, R. (1973). Leon Trotsky and the politics of economic isolation. Cambridge University Press.

Depretto, J. (1994). La formation de la classe ouvrière soviétique. Le Mouvement Social, 169, 47-65.

Dobb, M. (1967). The discussions of the 1920's about building socialism. Annali, 9, 136-166. Instituto Giangiacomo Feltrinelli.

Douds, L. (2018). Inside Lenin's government. Ideology, power and practice in the early Soviet State. Bloomsbury Publishing Plc.

Drobizhev, V.Z. (1979). Nekotorie voprosy izucheniia istorii sovetskogo rabochego klassa v sovetskoi istoriografii. Metodologicheskie i metodicheskie problemy izucheniia rabochego klassa sotsialisticheskogo obshchestva, 3-52.

Filtzer, D. (1993). Labour and the contradictions of Soviet planning under Stalin: The working class and the regime during the first years of forced industrialization. Critique: Journal of Socialist Theory, 20 (1), 71-103. DOI: $10.1080 / 03017609308413356$.

Fitzpatrick, S. (2007). Revisionism in Soviet history. History and Theory, 46 (4), 77-91.

Getty, J.A., y Naumov, O.V. (1999). The road to terror. Stalin and the self-destruction of the Bolsheviks, 1932-1939. Yale University Press.

Graziosi, A. (1991). Building the first system of State industry in history. Piatakov's VSNKh and the crisis of the NEP, 1923-1926. Cahiers $d u$ Monde Russe et Soviétique, 32 (4), 539-580.

Koenker, D. (1981). Moscow workers and the 1917 Revolution. Princeton University Press.

- (1989). Urbanization and deurbanization in the Russian Revolution and civil war. En D. Koenker, W. Rosenberg y R.G. Suny (eds.), Party, State, and society in the Russian civil war. Explorations in social history (pp. 81-104). Indiana University Press.

- (2005). Republic of Labor. Russian printers and Soviet socialism, 1918-1930. Cornell University Press.

Koenker, D., y Rosenberg, W. (1989). Strikes and Revolution in Russia, 1917. Princeton University Press.

Kotkin, S. (1995). Magnetic mountain. Stalinism as a civilization. University of California Press. 
- (1998). 1991 and the Russian Revolution: Sources, conceptual categories, analytical frameworks. The Journal of Modern History, 70 (2), 384-425.

Lenin, V.I. (1958). ¿Se sostendrán los bolcheviques en el poder? En Obras completas, t. XXVI (pp. 75-124). Cartago.

- (1960a) Las tareas inmediatas del poder soviético. En Obras completas, t. XXVII (pp. 317-346). Cartago.

- (1960b). Sobre el infantilismo de la "izquierda" y el espíritu pequeñoburgués. En Obras completas, t. XXVII (pp. 317-346). Cartago.

- (1960c). Discurso pronunciado en la conferencia de las organizaciones sin partido de la región de Blagushe-Lefortovo. En Obras completas, t. XXX (pp. 347-349). Cartago.

- (1960d). Carta a Stalin, 24 de enero de 1920. En Obras completas, t. XXX (pp. 297-299). Cartago.

- (1960e). El impuesto en especie (significación de la Nueva Política Económica y su ubicación). En Obras completas, t. XXXII (pp. 322-359). Cartago.

Lewin, M. (1985). More than one piece is missing in the puzzle. Slavic Review, 44 (2), 239-243.

Lih, L., Naumov, O., y Khlevniuk, O. (1995). Stalin's letters to Molotov. 19251936. Yale University Press.

Linhart, R. (1976). Lénin, les paysans, Taylor. Essai d'annalyse material historique de la naissance du systèm productif soviétique. Seuil.

Malle, S. (1985). The Economic organization of war communism, 1918-1921. Cambridge University Press.

Mandel, D. (2018). The Petrograd workers in the Russian Revolution. February 1917-June 1918 [1983]. Koninklijke Brill nv.

Marot, J.E. (2012). The October Revolution in prospect and retrospect. Koninklijke Brill nv.

Murphy, K. (2005). Revolution and counterrevolution. Class struggle in a Moscow metal factory. Berghahn Books.

- (2008). Strikes during the early Soviet period, 1922 to 1932: From working-class militancy to working-class passivity? En D. Filtzer et al. A Dream deferred. New studies in Russian and Soviet labour history (pp. 171-191). Peter Lang AG-International Academic Publishers.

Orlovsky, D. (1989). State building in the civil war era. The role of the lower-middle strata. En D. Koenker, W. Rosenberg y R.G. Suny (eds.), Party, State, and society in the Russian civil war. Explorations in social history (pp. 133-157). Indiana University Press.

- (1994). The hidden class: White-collar workers in the Soviet 1920s. En L. Siegelbaum y R.G. Suny (eds). Making workers Soviet. Power, class and identity (pp. 220-252). Cornell University Press.

Pirani, S. (2006). The party elite, the industrial managers and the cells: Early stages in the formation of the Soviet ruling class in Moscow, 1922-23. Revolutionary Russia, 2 (19), 197-228.

Pospielovsky, A. (1997). Strikes during the NEP. Revolutionary Russia, 1 (10), 1-34. 
Rassweiler, A.D. (1983). Soviet labor history of the 1920 s and the 1930 s. Journal of Social History, 17 (1), 147-158. DOI: 10.1353/jsh/17.1.147.

Rees, E.A. (1987). State control in Soviet Russia. The rise and fall of the workers' and peasants' inspectorate, 1920-34. Palgrave Macmillan.

Rezolyutsiya chetvertoy konferentsii profsoyuznykh liderov (1918). Professinalnyi Vestnik, 5-6, pp. 13-14.

Rosenberg, W.G. (1978). Workers and workers' control in the Russian Revolution. History Workshop Journal, 5 (1), 89-97. DOI: 10.1093/hwj/ 5.1.89.

- (1985). Reply. Slavic Review, 44 (2), 251-256.

- (1994). Social mediation and State construction(s) in Revolutionary Russia. Social History, 19 (2), 169-188.

Rossman, J. (2005). Worker resistance under Stalin. Class and Revolution on the shop floor. Harvard University Press.

Sánchez-Sibony, O. (2014). Red globalization. The political economy of the Soviet Cold War from Stalin to Khrushchev. Cambridge University Press.

- (2019). Global money and bolshevik authority: The NEP as the first socialist project. Slavic Review, 78 (3), 694-716.

Sgrazzutti, J., Oliva, A. (2017). Aportes para la comprensión del taylorismo soviético de Octubre a la NEP (1917-1929). Anuario de la Escuela de Historia, 29, 9-47. http://anuariodehistoria.unr.edu.ar/ojs/index.php/ Anuario/index.

Sirianni, C. (1985). Rethinking the significance of worker's control in the Russian Revolution. Economic and Industrial Democracy, 6 (1), 65-91. DOI: doi.org/10.1177/0143831X8561004.

Smith, S.A. (1985). Red Petrograd. Revolution in the factories, 1917-1918. Cambridge University Press.

Vorozheikin, I.E., y Seniavskii, S.L. (1977). Rabochii klass - vedushchaia sila sovetskogo obshchestva. Voprosy metodologii $i$ istoriografii.

Ward, C. (1990). Russia's cotton workers and the New Economic Policy: Shopfloor culture and State policy, 1921-1929. Cambridge University Press. 